\title{
Improved parameterization of Antarctic krill target strength models
}

\author{
Gareth L. Lawson, ${ }^{\text {a) }}$ Peter H. Wiebe, and Carin J. Ashjian \\ Biology Department, Woods Hole Oceanographic Institution, Woods Hole, Massachusetts 02543 \\ Dezhang Chu and Timothy K. Stanton \\ Applied Ocean Physics and Engineering Department, Woods Hole Oceanographic Institution, \\ Woods Hole, Massachusetts 02543
}

(Received 20 April 2005; revised 31 October 2005; accepted 2 November 2005)

\begin{abstract}
There are historical discrepancies between empirical observations of Antarctic krill target strength and predictions using theoretical scattering models. These differences are addressed through improved understanding of key model parameters. The scattering process was modeled using the distorted-wave Born approximation, representing the shape of the animal as a bent and tapered cylinder. Recently published length-based regressions were used to constrain the sound speed and density contrasts between the animal and the surrounding seawater, rather than the earlier approach of using single values for all lengths. To constrain the parameter governing the orientation of the animal relative to the incident acoustic wave, direct measurements of the orientation of krill in situ were made with a video plankton recorder. In contrast to previous indirect and aquarium-based observations, krill were observed to orient themselves mostly horizontally. Averaging predicted scattering over the measured distribution of orientations resulted in predictions of target strength consistent with in situ measurements of target strength of large krill (mean length $40-43 \mathrm{~mm}$ ) at four frequencies $(43-420 \mathrm{kHz})$, but smaller than expected under the semi-empirical model traditionally used to estimate krill target strength. () 2006 Acoustical Society of America. [DOI: 10.1121/1.2141229]
\end{abstract}

PACS number(s): 43.30.Sf, 43.20.Fn, 43.30.Ft [KGF]

Pages: $232-242$

\section{INTRODUCTION}

The Antarctic krill, Euphausia superba (henceforth referred to as "krill"), is a key species of marine zooplankton linking primary producers and higher predators in the Southern Ocean (Laws, 1985) and is also the subject of a commercial fishery (Ichii, 2000). Abundance surveys for krill stock assessments and ecological studies typically employ acoustic techniques, as acoustics offer the advantage of continuous surveying over large areas in a short period of time. In order to relate acoustic measurements of echo energy to biological quantities like absolute abundance, however, it is critical to understand the efficiency with which the krill scatter sound, expressed in terms of their target strength. The present work seeks to address certain discrepancies that have resulted between theoretical and empirical approaches to understanding krill target strength.

Most modern acoustic surveys for krill, including those conducted by the international Committee for the Conservation of Antarctic Marine Living Resources (CCAMLR; SCCAMLR, 1991), employ the semi-empirical target strength model of Greene et al. (1991). This model relates target strength at the common survey frequency of $120 \mathrm{kHz}$ linearly to the logarithm of krill length and was derived on a theoretical basis from empirical observations at $420 \mathrm{kHz}$ of a variety of crustacean taxa in an enclosure (Greene et al., 1989; Wiebe et al., 1990). Measurements of Antarctic krill

\footnotetext{
${ }^{\text {a)} E l e c t r o n i c ~ m a i l: ~ g l a w s o n @ w h o i . e d u ~}$
}

swimming freely in an enclosure (Foote et al., 1990) and in situ observations (Hewitt and Demer, 1991) have yielded estimates of krill target strength consistent with the Greene et al. (1991) model.

Substantial progress has been made in the theoretical, physics-based modeling of the target strength of fluidlike crustacean zooplankton such as krill (reviewed in Stanton and Chu, 2000). State-of-the-art models employ the distorted-wave Born approximation (DWBA) to estimate the scattering using a simplified description of the shape of the animal. Such an approach accounts for the fact that scattering is a complicated function of the animal's length, shape, orientation, and acoustic material properties, as well as the frequency being used. In the case of euphausiids (the order encompassing Antarctic krill), the shape has typically been modeled as some kind of deformed cylinder. Scattering is integrated along the lengthwise axis of the cylinder, taking into account the phase shift arising from deformation of this axis due to curvature and variations in cross-sectional radius (Chu et al., 1993; Stanton et al., 1993; McGehee et al., 1998; Stanton et al., 1998; Demer and Conti, 2003). Lavery et al. (2002) employed the DWBA to estimate scattering as the volume integral over a fully 3 -D representation of the animal derived from computerized tomography. Target strengths predicted by these theoretical models have been verified by tank observations of individual tethered animals at a variety of frequencies, animal sizes, and angles of orientation relative to the incident acoustic wave. 
Although theoretical predictions are mostly consistent with tank-based measurements where the exact size, shape, and angle of orientation of the animal are known, problems have arisen in parameterizing the models in such a way that their predictions are consistent with the Greene et al. (1991) relationship, while keeping the parameters within biologically plausible ranges (Demer and Conti, 2003, 2005). In particular, the greatest uncertainty has surrounded the parameters governing the orientation of the animal and its acoustic material properties. As an individual krill goes from a horizontal to vertical orientation, its target strength as observed by a vertically aimed echosounder decreases by two or more orders of magnitude (Stanton et al., 1998; McGehee et al., 1998). Similarly, it has long been recognized that for bodies filled with fluid similar to the surrounding medium, target strength is highly sensitive to small changes in the contrasts between the sound speed and density within the body and those of the medium (i.e., the "acoustic material properties") (Anderson, 1950; Johnson, 1977; Holliday and Pieper, 1980; Greenlaw and Johnson, 1982; Chu et al., 2000).

In order to make field-applicable predictions of target strength, it is thus highly important to constrain properly these parameters governing orientation and acoustic material properties, but very little information exists concerning their natural distribution. Chu et al. (1993) and Demer and Conti (2005) have estimated krill orientation indirectly from measurements of volume backscattering and target strength, respectively, but no direct and quantitative measurements exist of krill in situ orientation. Acoustic material properties are typically assumed to be uniform within the animal's body and constant with respect to animal length, although Chu and Wiebe (2005) have shown that in the Antarctic krill both the sound speed and density contrasts are significantly related to length. When McGehee et al. (1998) used the then bestavailable observations made by Kils (1981) of krill orientation in an aquarium and by Foote (1990) of krill acoustic material properties to parameterize a DWBA-based scattering model, their predictions of krill target strength were ca. $6 \mathrm{~dB}$ lower than predicted by the Greene et al. (1991) semiempirical relationship.

Motivated by these discrepancies between the predictions of theoretical scattering models and the Greene et al. relationship, we seek to improve model parameterization. Backscattering from individual krill is predicted using the DWBA, representing the shape of the animal as a uniformly bent and smoothly tapered cylinder. Improved parameterization is achieved by making direct observations of krill in situ orientation with a video plankton recorder (VPR; Davis et al., 1992). In contrast to previous studies where single values of the acoustic material properties have been used for all lengths of krill, we also apply Chu and Wiebe's (2005) length-based regressions of krill sound speed and density contrasts. We further assess the validity of this parameterization by making in situ observations of krill target strength at four frequencies.

\section{METHODS}

\section{A. Theoretical krill scattering model}

The scattering model employed here is the DWBAbased deformed cylinder model with homogeneous acoustic material properties first used by Chu et al. (1993) and Stanton et al. (1993) and expressed more explicitly in Stanton et al. (1998). The general formulation of the DWBA gives the far-field scattering amplitude in the backscatter direction $\left(f_{\mathrm{bs}}\right)$ for a body of finite-length as an integral over the body's volume (Morse and Ingard, 1968). The DWBA assumes that the contrasts between the speed of sound and density within the body and the surrounding seawater are small (i.e., weakly scattering bodies) and that the body has negligible elastic properties, thereby not supporting shear waves (i.e., fluidlike).

Under the assumption that the shape of the animal can be approximated as a deformed cylinder (elongated and circular in cross section), Stanton et al. (1998) showed that the volume integral of the general DWBA formulation can be reduced to a line integral along the cylinder's lengthwise axis. Various kinds of cylinders have been used to represent the krill's irregular shape, ranging through a progression of complexity including straight, smoothly tapered, uniformly bent, and randomly rough cylinders, as well as the case where nonuniform variations in cross-sectional radius are used to represent appendages (Stanton and Chu, 2000). We choose to model the krill's shape as a uniformly bent and smoothly tapered cylinder. This representation only coarsely captures the actual shape of the animal; additional justification for not using a higher resolution shape description is provided in the discussion.

In the case of a uniformly bent cylinder with radius of curvature $\rho_{c}$, Stanton et al. (1998) give the expression for the scattering amplitude as

$$
\begin{aligned}
f_{\mathrm{bs}}= & \frac{k_{1} \rho_{c}}{4} \mathrm{e}^{\mathrm{i} 2 k_{2} \rho_{c}} \int a\left(\gamma_{\kappa}-\gamma_{\rho}\right) \mathrm{e}^{-\mathrm{i} 2 k_{2} \rho_{c} \cos \beta_{\text {tilt }}} \\
& \times \frac{J_{1}\left(2 k_{2} a \cos \beta_{\text {tilt }}\right)}{\cos \beta_{\text {tilt }}} \mathrm{d} \beta_{\text {tilt }},
\end{aligned}
$$

where $k$ is the acoustic wave number in the surrounding seawater (subscript 1) and the body (subscript 2); $a$ is the crosssectional radius of the cylinder; $\gamma_{\kappa}$ and $\gamma_{\rho}$ are related to the densities $(\rho)$ and sound speeds $(c)$ of the surrounding seawater (1) and the body (2) following $\gamma_{\kappa}=\left(\kappa_{2}-\kappa_{1}\right) / \kappa_{1}, \gamma_{\rho}=\left(\rho_{2}\right.$ $\left.-\rho_{1}\right) / \rho_{2}$, and $\kappa=\left(\rho c^{2}\right)^{-1}$, where $\kappa$ is the compressibility; $J_{1}$ is the Bessel function of the first kind of order one; and $\beta_{\text {tilt }}$ is the angle between the incident wave $\left(\mathbf{k}_{\mathrm{i}}\right)$ and the cross section of the cylinder at each point along its axis (Stanton et al., 1998).

To accommodate further the actual shape of the krill, the ends of the cylinder are tapered by making the radius a function of position along the lengthwise axis $(z)$ :

$$
a(z)=a_{0} \sqrt{1-\left(\frac{z}{L / 2}\right)^{T}},
$$

where $a_{0}$ is the radius of the cylinder at its mid-point, $T$ is a parameter controlling how quickly the cylinder tapers, and $L$ 
TABLE I. Definitions of standard lengths (SL) for krill, from Mauchline (1980). Different lengths were required for the various purposes of the present study due to differences in how previous workers have defined krill length.

\begin{tabular}{|c|c|c|c|}
\hline Name & Definition & Use in the present work & $\begin{array}{l}\text { Relation } \\
\text { to SL3 }\end{array}$ \\
\hline SL 1 & $\begin{array}{l}\text { Anterior tip of rostrum to } \\
\text { posterior end of uropods }\end{array}$ & $\begin{array}{l}\text { Length used in sound speed and density } \\
\text { contrast regression equations }\end{array}$ & $1.236 \times$ SL3 \\
\hline SL 2 & $\begin{array}{l}\text { Anterior of eye to end of sixth } \\
\text { abdominal segment }\end{array}$ & $\begin{array}{l}\text { Length of the equivalent cylinder used to } \\
\text { represent the krill in modeling target strength }\end{array}$ & $1.069 \times$ SL3 \\
\hline SL 3 & $\begin{array}{l}\text { Posterior base of eye stalk to end } \\
\text { of sixth abdominal segment }\end{array}$ & $\begin{array}{l}\text { Length measured in silhouette analysis of } \\
\text { MOCNESS catches }\end{array}$ & $\cdots$ \\
\hline
\end{tabular}

is the cylinder's length with $z=0$ the animal's midpoint (Chu et al., 1993, who set $\mathrm{T}=10$ ).

Approximate solutions can be found for limiting expressions of Eq. (1) with respect to wavelength, but, more typically, the cylinder is discretized into a series of thin diskshaped differential elements and the integral performed numerically.

The differential backscattering cross section $\left(\sigma_{\mathrm{bs}}\right)$ is defined as the square of the magnitude of the backscattering amplitude, and target strength (TS) is simply $\sigma_{\mathrm{bs}}$ in decibel form $\left(\mathrm{dB}\right.$ relative to $\left.1 \mathrm{~m}^{2}\right)$ :

$$
\mathrm{TS}=10 \log \sigma_{\mathrm{bs}}=10 \log \left|f_{\mathrm{bs}}\right|^{2} .
$$

\section{B. Model parameterization}

Predictions of target strength using the above model are clearly dependent on a variety of parameters, including those governing the animal's shape $\left(L, a_{0}, T\right.$, and $\left.\rho_{c}\right)$, its acoustic material properties $\left(\gamma_{\kappa}\right.$ and $\left.\gamma_{\rho}\right)$, and its orientation $\left(\beta_{\text {tilt }}\right)$. As indicated above, the parameters $\gamma_{\kappa}$ and $\gamma_{\rho}$ are themselves functions of the sound speed and density contrasts between the animal and the surrounding medium $\left(h=c_{2} / c_{1}\right.$ and $g$ $=\rho_{2} / \rho_{1}$, respectively). The emphasis here is on properly constraining the key parameters of krill orientation and acoustic material properties.

Target strength was therefore predicted on the basis of various combinations of orientation and sound speed and density contrast values. Predictions at a frequency of $120 \mathrm{kHz}$ were made for cylinder lengths of 4 to $70 \mathrm{~mm}$, in 1 -mm increments. Predictions were also made holding length constant at $43.3 \mathrm{~mm}$, for frequencies of $5-500 \mathrm{kHz}$, in $5-\mathrm{kHz}$ increments.

The equivalent cylinder used to represent the krill's shape was defined on the basis of the animal's average radius $\left(a_{0}\right)$ and length $(L)$, defined as standard length 2 of Mauchline (1980) (Table I), following the approach of Stanton and Chu (2000). Other than length, the shape parameters were held constant for all simulations: a slight taper parameter $T$ of 10 was used, and the cross-sectional radius of the cylinder at its mid-section was related to length via $a_{0}$ $=L / 18.4$. This constant was derived by measuring the length and average radius (averaged over ten measurements along the animal's length) of 50 preserved krill captured with nets (see below). The radius of curvature was taken to be $\rho_{\mathrm{c}}$ $=3 \mathrm{~L}$, based on measurements of 50 randomly chosen krill observed with the video plankton recorder, but note that backscattering cross sections averaged over a range of angles of orientation (as is done here, see below) are mostly independent of the cylinder's bend, for $\rho_{\mathrm{c}} \geqslant 2 L$ (Stanton et al., 1993).

\section{Sound speed and density contrasts}

Chu and Wiebe (2005) showed that the sound speed and density contrasts of Antarctic krill are significantly related to animal length. The $g$ and $h$ values used to parameterize the scattering model were therefore estimated from their regression equations:

$$
\begin{aligned}
& g=5.439 \times 10^{-4} L(\mathrm{~mm})+1.002, \\
& h=4.981 \times 10^{-4} L(\mathrm{~mm})+1.009,
\end{aligned}
$$

where length $(L)$ is standard length 1 (Table I). The acoustic material properties were assumed not to vary within the animal, and so single values for each of these parameters were calculated for each krill length examined. For comparison, predictions of target strength were also made based on the length-invariant krill material property measurements of Foote $(1990)(g=1.0357, h=1.0279)$. The minimum size of animal examined by Chu and Wiebe (2005) was $25 \mathrm{~mm}$. Extrapolating for lengths smaller than this increasingly produced implausibly small estimates of $g$ and $h$. The material properties estimated from the regressions for a 25 mm-long animal therefore were used for lengths smaller than $25 \mathrm{~mm}$. Note that the Chu and Wiebe (2005) measurements were made on krill collected in the same study area and at the same time of year (austral fall) as the empirical observations of krill orientation and target strength described below, but a year later (2002).

\section{Animal orientation}

Equation (1) allows the scattering amplitude to be predicted for an individual animal of a given length at a single angle of orientation $(\theta)$, defined as the angle between the line joining the bent cylinder's ends and the horizontal plane. Assuming a vertically aimed echosounder, an animal oriented horizontally in the water $\left(\theta=0^{\circ}\right)$ is at normal acoustic incidence. At the cylinder's mid-point, the relationship between $\beta_{\text {tilt }}$ and $\theta$ is simply $\beta_{\text {tilt }}=\theta$, while elsewhere along the cylinder's axis, it varies due to the cylinder's curvature.

In linear echo-integration theory, the echoes from individual animals within the acoustic beam are assumed to sum incoherently to yield measurements of volume backscattering. In order to simulate the averaging over ensembles of many individuals that occurs during echo-integration sur- 


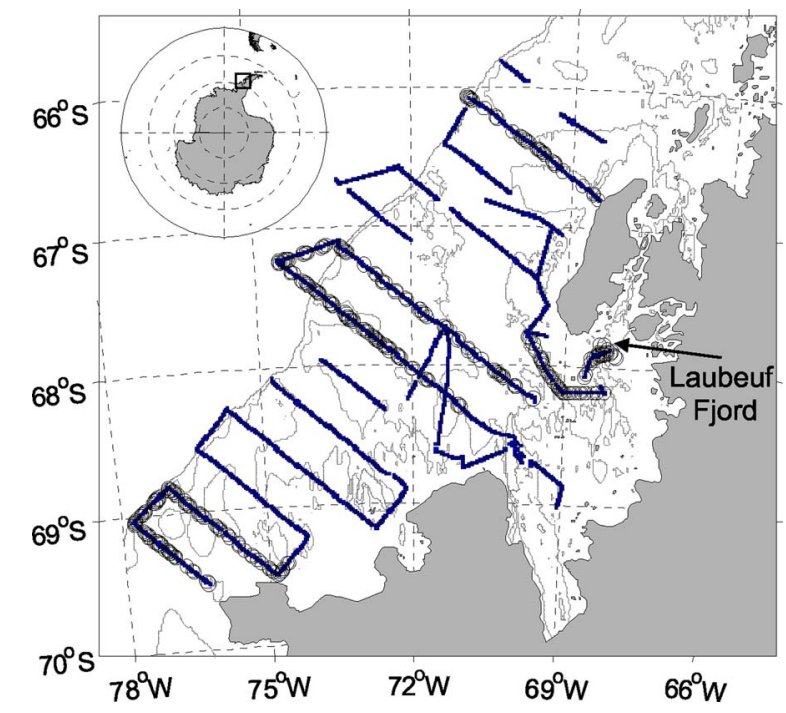

FIG. 1. Study site, covering a region of the continental shelf west of the Western Antarctic Peninsula. Black lines show survey transects along which acoustic, video, and environmental data were collected. Circles indicate the subsections of these lines where video images of krill were captured and analyzed. Contours show the 450- and 1000-m isobaths. Laubeuf Fjord is the region where direct measurements were made of krill target strength and where two net tows sampled acoustically identified krill patches.

veys, average scattering for each krill length investigated was calculated over a probability density function of angles of orientation $[w(\theta)]$, following

$$
\bar{\sigma}_{\mathrm{bs}}=\int_{\theta} \sigma_{\mathrm{bs}}(\theta) w(\theta) \mathrm{d} \theta .
$$

Average target strength was then defined as the decibel form of $\bar{\sigma}_{\mathrm{bs}}$.

Average scattering was calculated in this way for the observed probability density function of angles of orientation described below. For comparison, average scattering was also calculated over the normal distribution of orientations observed in an aquarium by Kils (1981), $N\left(\bar{\theta}, \sigma_{\theta}\right)$ $=N\left(45.3^{\circ}, 30.4^{\circ}\right)$, where $\bar{\theta}$ is the mean angle of orientation and $\sigma_{\theta}$ is the associated standard deviation. Similarly, for some comparisons, a length-averaged predicted scattering was calculated at each length under investigation by averaging over a distribution of neighboring animal lengths.

\section{Empirical approach}

Video, acoustic, and environmental data were collected from the RVIB N.B. Palmer in April-June of 2001, as part of the U.S. Southern Ocean GLOBal ECosystems Dynamics program (GLOBEC; Hofmann et al., 2002). The study site was a continental shelf region west of the Western Antarctic Peninsula (Fig. 1). All data were collected with the BIoOptical Multi-frequency Acoustical and Physical Environmental Recorder (BIOMAPER-II; Wiebe et al., 2002), a towed system consisting of a multi-frequency echosounder with both up- and down-looking transducers, a video plankton recorder (VPR), and an environmental sensor package (conductivity, temperature, and depth sensor; fluorometer; transmissometer). The BIOMAPER-II was "towyoed" obliquely up and down through the water column between 20-
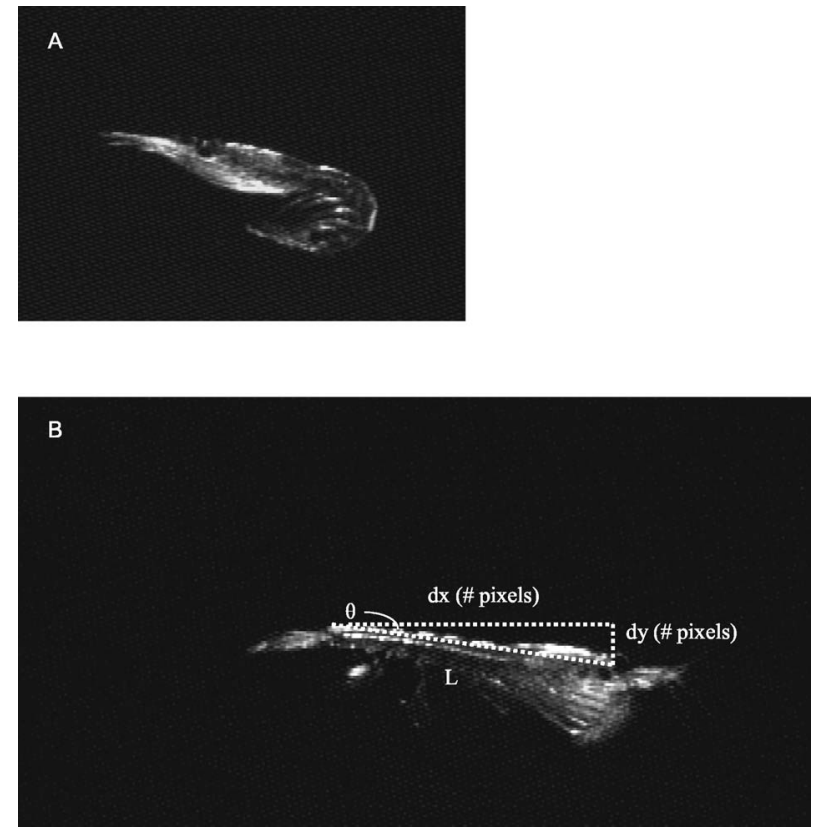

FIG. 2. Representative krill images. (a) A typical krill exhibiting the tail-flip escape response. (b) A krill of length $11.1 \mathrm{~mm}$ oriented at $-9.4^{\circ}$ relative to the horizontal. The horizontal $(d x)$ and vertical $(d y)$ excursions from the animal's tail to its eye were measured in pixels. Knowing the size of the field of view in both pixels and distance, the animal's orientation relative to horizontal $(\theta)$ and length $(L)$ were then calculated.

and 300-m depth as the vessel proceeded along the track-line between stations at 4 to 6 knots, and surveying was conducted around the clock. Data were collected along 13 transect lines running across the continental shelf and perpendicular to the Peninsula coastline; subsections of these lines were selected for analysis of krill orientation (Fig. 1).

\section{Measuring the in situ orientation of krill}

Measurements of krill in situ orientation were made directly from still digital images captured from video collected with the VPR, in a similar manner to Benfield et al. (2000). The VPR consisted of a camera and 16-W strobe mounted on the towbody forward of the tow point, separated by $0.5 \mathrm{~m}$, and aimed towards one another and perpendicular to the direction of the body's motion. The field of view of the camera was calibrated using a translucent grid placed in the center of focus and was found to be $31 \times 24.5 \mathrm{~mm}$ (width by height). The camera sampled at a rate of $60 \mathrm{~Hz}$, synchronized to the strobe. Video fields were time stamped and digitized at a resolution of 640 by 207 pixels. Regions of each field that were in focus were automatically extracted and saved as tagged image file format (tif) images [see Davis et al. (1996) for additional details].

These images were then visually examined and only images that were definitely krill, where the animal's whole body was in the frame and the image was in focus, were used for further analysis. In response to vigorous disturbances, krill are known to perform a rapid tail-flip response (O’Brien, 1987). Animals performing such a tail-flip often were captured in video images [Fig. 2(a)]; such images were excluded from analysis.

For the remaining images, the horizontal $(d x)$ and verti- 
cal $(d y)$ excursions in pixels from the tail of each krill to its eye were measured [Fig. 2(b)] and the animal's angle of orientation $(\theta)$ calculated as

$$
\theta=\tan ^{-1}\left(d y \frac{\text { fov height } \mathrm{mm}}{\text { fov height pixels }} / d x \frac{\text { fov width } \mathrm{mm}}{\text { fov width pixels }}\right),
$$

where fov denotes the field of view. Length $(L)$ was calculated via the Pythagorean theorem. An animal oriented perfectly horizontal was defined as being at an angle of $0^{\circ}$ and one oriented belly-up as $180^{\circ}$, with positive angles indicating a head upwards tilt.

In order to use the horizontal and vertical extent of the animal from the image to calculate orientation relative to the horizontal, only images where the krill was perpendicular to the direction faced by the camera, and where the animal was in side-view, were analyzed (Benfield et al., 2000). Identifying animals in side-view was done by looking for overlap of the eyes and inspecting the legs. Determining whether animals were plane to the camera was done by visually assessing the ratio of the vertical to horizontal extent of different segments of the animal.

To confirm that the detection and extraction of krill images by the VPR system were not biased against any particular angles of orientation due to insufficient illumination or focus level, segments of the raw videotapes were examined and the intensity and focus level of extracted krill images were analyzed in relation to the measured angle of orientation. No such bias was evident.

These measurements of krill orientation relative to the reference frame of the camera then had to be corrected for the pitch of the towed body in order to give the orientation relative to true horizontal. Data were collected on the pitch of the BIOMAPER-II every $5 \mathrm{~s}$. To capture the gross behavior of the body while reducing error associated with highfrequency variability, the pitch data were subjected to a tenpoint median filter. The filtered pitch observation made nearest in time to each image was then used to correct the measured angles of orientation. Corrections were also made based on interpolations of the raw and median-filtered pitch data, but the resulting distributions of orientations differed little from the previously described correction protocol. Since the body's pitch was especially variable when the vessel was on station, only images collected while the vessel was moving along survey transects were considered.

\section{Measuring the in situ target strength of krill}

Measurements of acoustic target strength were made at frequencies of $43,120,200$, and $420 \mathrm{kHz}$, for comparison with theoretical predictions. All transducers were circular and split-beam, with $3^{\circ}$ half-power beamwidths, other than the $7^{\circ}$-wide $43-\mathrm{kHz}$ transducers. Each transducer was acoustically calibrated by the manufacturer (Hydroacoustic Technologies Inc., Seattle, WA, USA) prior to the cruise for source level, receive sensitivity, electro-mechanical "stiffness" (used to determine the position of a target within the split-beam), and transmit and receive beam patterns. An in situ calibration with a $38-\mathrm{mm}$ tungsten carbide ( $6 \%$ cobalt) standard target also was performed during a cruise later that year. A $10-\mathrm{kHz}$ bandwidth chirp pulse was used, with an effective pulse duration of $0.18 \mathrm{~ms}$, and a ping rate of 0.3 pings $\mathrm{s}^{-1}$. The system's dynamic range allowed target strength data to be collected between -100 and $-40 \mathrm{~dB}$. Profiles of noise levels (ship's noise, ambient noise, and system noise combined) versus depth were made in situ near the start of each cruise. Target strength measurements smaller than these noise levels were not recorded. Information on the target's location within the beam from split-beam analysis was used to remove the effects of beam pattern. In order to reduce the likelihood that multiple targets were mistakenly accepted as individual target strengths, only measurements made at a beam pattern factor (an indicator of off-axis position) between 0 and $-3 \mathrm{~dB}$, where the length of the received acoustic pulse at half-power was within $12.5 \%$ of the transmitted pulse, and at a range of less than $13 \mathrm{~m}(8 \mathrm{~m}$ for the $43 \mathrm{kHz}$ ) were included for analysis. Densities of krill larger than $15 \mathrm{~mm}$ in the aggregations were estimated to be ca. 5 individuals $\mathrm{m}^{-3}$, and so the selected maximum ranges limited observations to cases where on average there was less than one animal per ensonified volume.

Measurements of target strength were made continuously over the course of the survey. Due to uncertainties in associating particular target strength observations with particular taxa, the focus here is on measurements made in Laubeuf Fjord at the end of the cruise (Fig. 1). Large patches of enhanced volume backscattering were present in this location (Lawson et al., 2004; Wiebe et al., 2004). Using a 1 $-\mathrm{m}^{2}$ Multiple Opening/Closing Net and Environmental Sensing System (MOCNESS; Wiebe et al., 1985), eight discrete samples were collected through these patches at depths between 50 and $100 \mathrm{~m}$, at each of two closely separated tow locations within the fjord (tow numbers M21 and M22; Wiebe et al., 2004). Both net catches and VPR observations confirmed that these acoustically observed patches were composed almost exclusively of krill. The net catches provide an estimate of the length distribution of the krill in these patches, allowing the observations of target strength to be associated with a particular length range of krill. Krill lengths were measured for an aliquot of each net sample using the silhouette method of Davis and Wiebe (1985) as standard length 3 and multiplied by constant scaling factors to arrive at the lengths used for modeling target strength and estimating $g$ and $h$ (Table I).

\section{RESULTS}

\section{A. In situ observations of krill orientation}

In total, the orientations of 972 individual krill were measured. The median and mean of the entire distribution of measured angles, for all lengths of krill observed combined, were $-0.5^{\circ}$ and $9.7^{\circ}$, respectively, with a standard deviation of $59.3^{\circ}$ (Fig. 3). Defining the dominant mode as all observations between $-100^{\circ}$ and $100^{\circ}$, the median and mean of this mode were $-3.4^{\circ}$ and $0^{\circ}$, respectively, with a standard deviation of $27.3^{\circ}$ (Fig. 3). Two smaller modes also were evident, centered near $140^{\circ}$ and $-160^{\circ}$.

Between day (0900-1500 h) and night (1700-0700 h), the central mode shifted from slightly above $0^{\circ}$ to slightly below ( $t$ test for day/night differences $t=6.02, p<1 \times 10^{-8}$ ). 


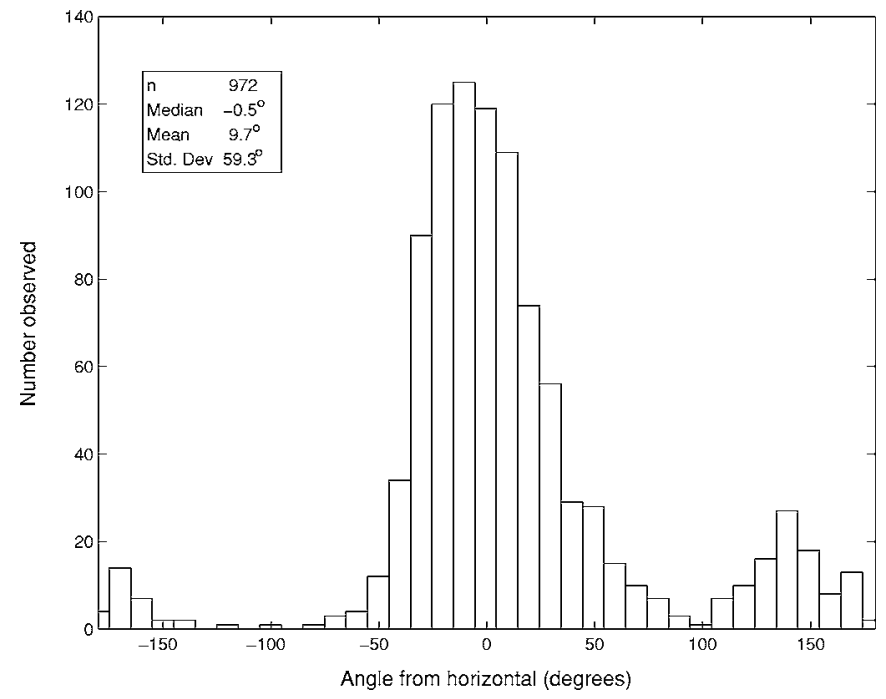

FIG. 3. Frequency distribution of angles of orientation for all lengths of krill combined, after correction for the pitch of the towed body. The median and mean of the entire distribution were $-0.5^{\circ}$ and $9.7^{\circ}$, respectively, with a standard deviation of $59.3^{\circ}$. Defining the central mode as all observations between $-100^{\circ}$ and $100^{\circ}$, the median and mean of this mode were $-3.4^{\circ}$ and $0^{\circ}$, respectively, with a standard deviation of $27.3^{\circ}$.

More observations were made by night (625) than by day (211), perhaps explaining why the distribution over all measurements was slightly negative. The distribution of observed orientations broadened with estimated krill length for lengths between 4 and $6 \mathrm{~mm}$ and then narrowed for greater lengths (Fig. 4). At estimated lengths greater than $6 \mathrm{~mm}$, the smaller modes near $140^{\circ}$ and $-160^{\circ}$ were no longer evident.

\section{B. Scattering model predictions}

Averaging scattering predictions from the DWBA-based deformed cylinder model over this VPR-derived distribution of angles of orientation following Eq. (6) resulted in higher average target strengths at $120 \mathrm{kHz}$ relative to krill length than with the Kils (1981) distribution (Fig. 5). Although scattering is a complex function of animal length, shape, orientation, material properties, and frequency, we choose to plot target strength in relation to length (standard length 2) as this

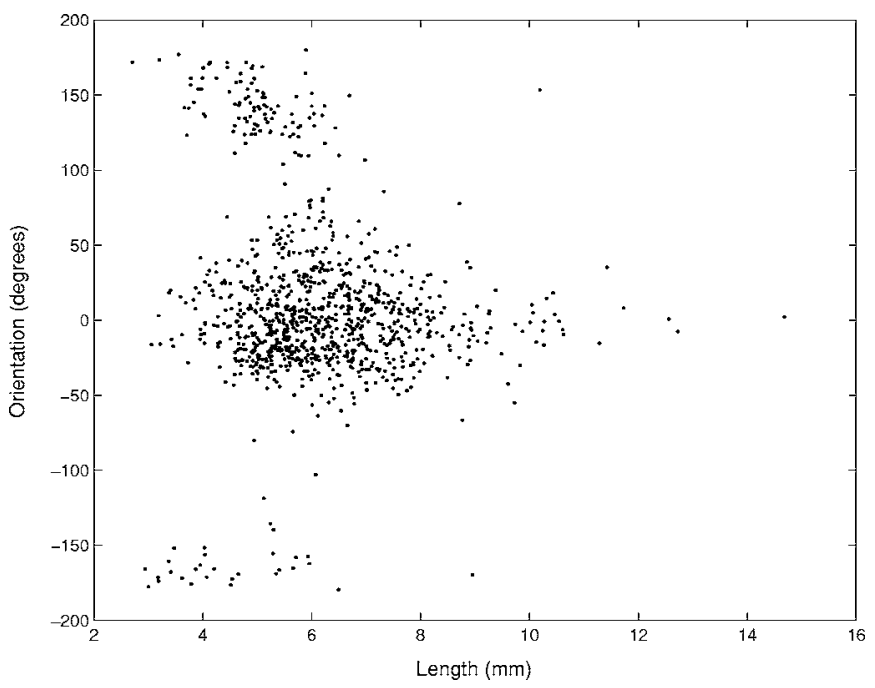

FIG. 4. Measured orientations in relation to krill length (mm).

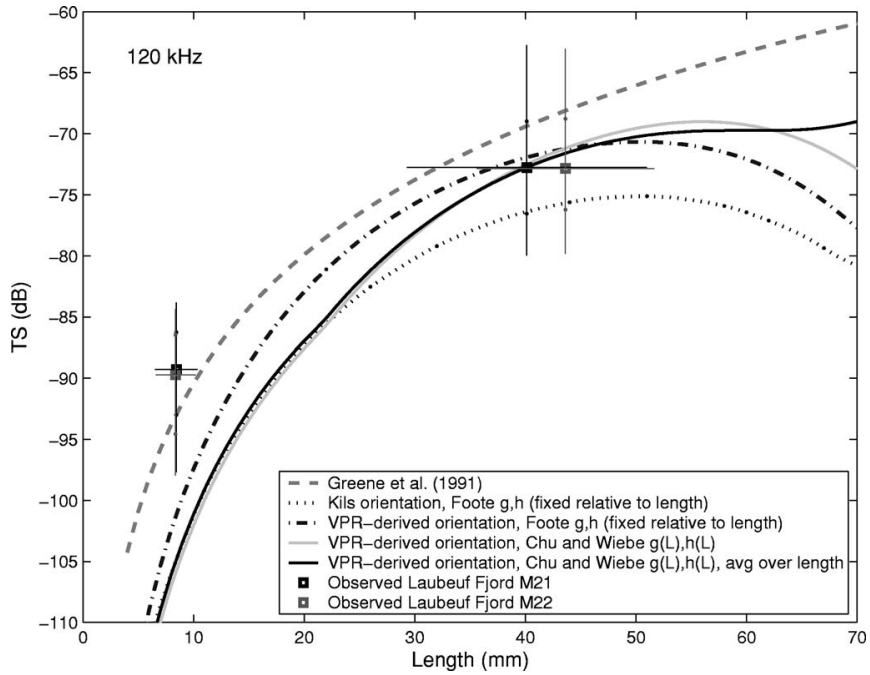

FIG. 5. Krill target strength ( $\overline{\mathrm{TS}})$ at $120 \mathrm{kHz}$ in relation to length (mm; SL 2 in Table I), averaged over orientation. Dashed gray line shows the Greene et al. (1991) and SC-CCAMLR (1991) empirical regression line TS= $-127.45+34.85 \log _{10}$ (length in $\mathrm{mm}$ ), where length is SL 1. All other lines indicate different parameterizations of the theoretical DWBA-based bent cylinder model, involving various combinations of the Foote (1990) lengthinvariant sound speed $(h)$ and density $(g)$ contrast measurements, the Chu and Wiebe (2005) $g$ and $h$ vs length $(L)$ regressions, Kils' (1981) aquarium observations of krill orientation, and the present VPR-derived in situ orientation measurements. The solid black line indicates predicted scattering averaged over a distribution of lengths (standard deviation $=15 \%$ of the mean). Squares show median in situ measurements of krill target strength made in Laubeuf Fjord relative to the mean length of krill sampled at the same depths and locations as the two net tows (M21 and M22 as black and gray squares, respectively). Vertical lines show 10th and 90th percentiles of target strength measurements; dots show the 25th and 75th percentiles. Horizontal lines represent one standard deviation from the mean length.

is the parameter most familiar to biologists and most relevant to ecological studies. These predictions were made with Foote's (1990) single values for the sound speed and density contrast parameters for all krill lengths and with averaging only over orientation and not over length. Note also that the VPR-derived orientation distribution observed for krill of length $3-15 \mathrm{~mm}$ is being applied to a broader range of lengths $(4-70 \mathrm{~mm})$. No difference was evident in model predictions for the daytime distribution of orientations as compared to that measured at night (not shown).

When the length-based regressions of Chu and Wiebe (2005) were used to estimate the material properties for each length examined, and these parameters were used in combination with the VPR-derived distribution of orientations, modeled target strengths were smaller than with the Foote (1990) values for lengths below $43 \mathrm{~mm}$ but larger for animals above this length. To simulate further the averaging over ensembles of individuals that occurs during echointegration surveys, length-averaged predicted scattering at each length under investigation was calculated over a normal distribution of neighboring animal lengths with a standard deviation of $15 \%$ of the mean (corresponding to the observed length variability from net tow M22). This resulted in a smoothing of the null in the target strength versus length relationship beyond $55 \mathrm{~mm}$ (Fig. 5).

In comparison to the Greene et al. (1991) semi-empirical target strength model, the present model parameterized with Chu and Wiebe's (2005) material property relationships and 

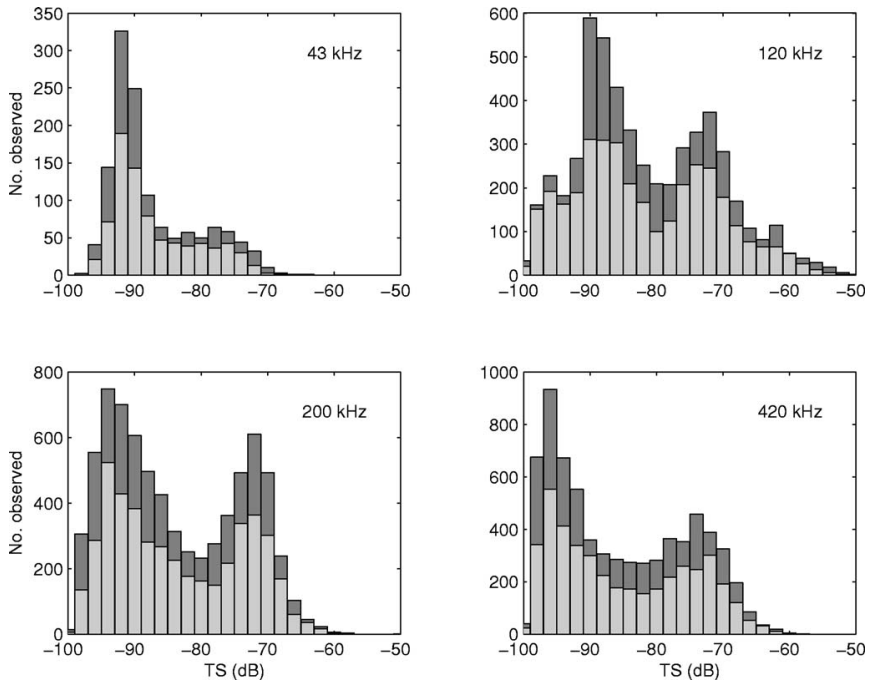

FIG. 6. Frequency distributions of in situ observed target strengths at 43, 120, 200, and $420 \mathrm{kHz}$ measured at the two net tow locations in Laubeuf Fjord (M21 and M22 indicated by dark and light gray bars, respectively).

the VPR-derived orientation distribution resulted in lower target strength predictions for all krill lengths, particularly for animals smaller than $25 \mathrm{~mm}$ and larger than $55 \mathrm{~mm}$.

\section{Model verification with empirical in situ target strength observations}

In situ observations of target strength within the acoustically observed patches in the vicinity of the two net tow locations in Laubeuf Fjord were bimodal at all four frequencies employed here (Fig. 6). The length distributions of krill sampled in the two net tows were similarly bimodal [see appendices in Wiebe et al. (2004)], allowing the small and large modes of the target strength distributions to be associated with the corresponding modes evident in the length distributions. The small and large modes of the length distribution from tow M21 had means of 8.4 and $40.5 \mathrm{~mm}$, respectively, and for tow M22 were 8.4 and $43.3 \mathrm{~mm}$. For both tows, the standard deviations of length were $22 \%$ and $15 \%$ of the mean, for the small and large modes, respectively.

Determining the central tendencies for the target strength modes was less straightforward, since the left-hand tail of the smaller mode was cut of by the system's threshold of $-100 \mathrm{~dB}$, while the right-hand tail of the smaller mode overlapped with the left-hand tail of the larger one (Fig. 6). Such issues of overlap and thresholding are well appreciated (Foote et al., 1986). For simplicity, the krill target strengths were assumed to be Rayleigh distributed, even though it is known that this is often not the case (Stanton et al., 2004). A Rayleigh distribution was fit to the smaller mode and used to extrapolate the target strength distributions below the -100 - $\mathrm{dB}$ threshold and above the point where overlap began with the larger mode $(-83 \mathrm{~dB}$ at $43 \mathrm{kHz}$, and $-80 \mathrm{~dB}$ at 120, 200, and $420 \mathrm{kHz}$ ); the larger mode distribution was similarly extrapolated below this point of overlap. Following extrapolation, the median of each target strength mode was calculated. The magnitude of the difference between the medians of the original truncated data and the extrapolated data never exceeded $1 \mathrm{~dB}$.

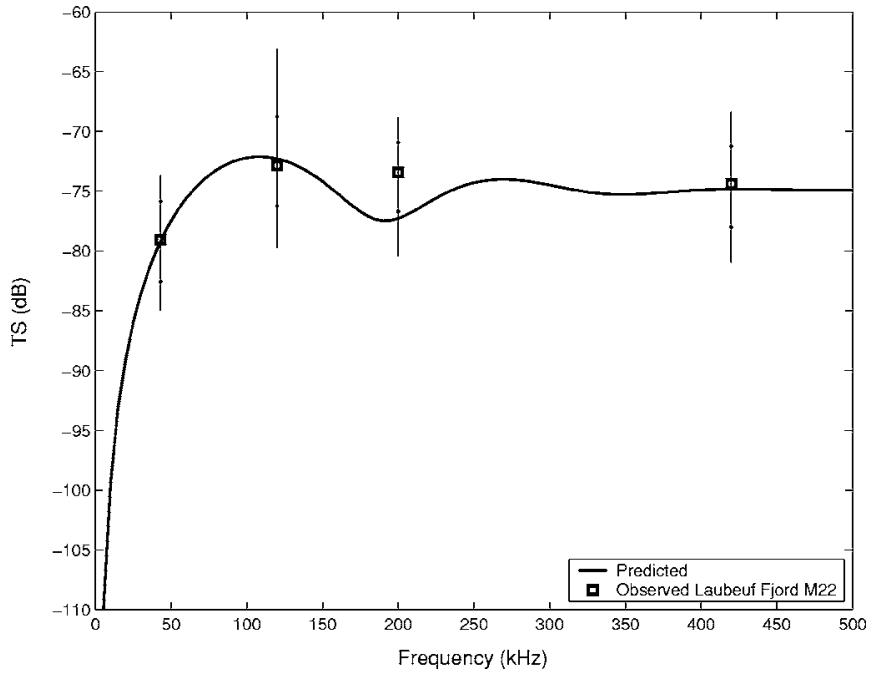

FIG. 7. Average target strength in relation to acoustic frequency $(\mathrm{kHz})$. The solid line shows the predictions from the DWBA bent cylinder model parameterized with the Chu and Wiebe (2005) material property relationships and the VPR-derived orientation distribution. Predicted differential backscattering cross sections were averaged over a normal length distribution with mean $43.3 \mathrm{~mm}$ and a standard deviation $=15 \%$ of the mean, corresponding to the observed length distribution from tow M22. Squares indicate median observed target strengths at the four BIOMAPER-II frequencies $(43,120,200$, and $420 \mathrm{kHz})$ in the vicinity of tow M22. Vertical lines show the 10th and 90th percentiles of observed target strength; dots show the 25th and 75 th percentiles.

After extrapolation, the median of the larger target strength mode at $120 \mathrm{kHz}$ was found to be $-72.8 \mathrm{~dB}$ in the vicinity of both net tow locations. This median target strength for the sampled length range is consistent with our newly parameterized target strength model (Fig. 5). We consider the median rather than the mean of the target strength distributions in order to reduce any potential bias towards higher values due to erroneous acceptance of multiple targets. The smaller mode of the $120-\mathrm{kHz}$ target strength distribution was centered at $-89.3 \mathrm{~dB}$ for tow M21 and $-89.7 \mathrm{~dB}$ for tow M22 (Fig. 5).

The DWBA bent cylinder model parameterized with the VPR-derived distribution of orientations and the Chu and Wiebe (2005) material property relationships was also used to predict the target strengths at increasing frequencies for a normal distribution of animals with mean length $43.3 \mathrm{~mm}$ and a standard deviation of $15 \%$. Median target strengths after extrapolation for the larger modes of our direct measurements of target strength at all four frequencies were generally consistent with the theoretical predictions (Fig. 7). The measurements at $200 \mathrm{kHz}$ compared less favorably to the predictions, likely due to error associated with the transducers at this frequency being calibrated less exhaustively than the others.

\section{DISCUSSION}

Krill observed in this study were found to orient themselves in a mostly horizontal fashion. This corresponds to normal acoustic incidence relative to a standard vertically aimed echosounder. When applied in conjunction with the length-based sound speed and density contrast relationships of Chu and Wiebe (2005), the observed distribution of orientations produced target strength predictions from a theoret- 
ical DWBA-based scattering model that are consistent with in situ observations of large krill target strength, but smaller than expected from the semi-empirical model of Greene et al. (1991).

Very few previous studies have examined directly the orientation of Antarctic krill or other euphausiids. Based on the qualitative observations of divers, Hamner et al. (1983) reported that schooling krill are always aligned uniformly and horizontally within aggregations, and that even when ascending or descending they orient at no more than a $5^{\circ}$ to $10^{\circ}$ angle. Similar to the present study, Kristensen and Dalen (1986) used underwater photography to measure the orientation of euphausiids (Meganyctiphanes norvegica and Thysanoessa spp.) in two Norwegian fjords and found that the mean orientation changed from slightly positive by night to slightly negative during the day. In contrast, observations made with a camera of the in situ orientation of similar euphausiid species in the Gulf of St. Lawrence revealed a shift in mean orientation from $27^{\circ}$ at 1400 to $51^{\circ}$ at 0200 , albeit with fairly high variability about this trend (Sameoto, 1980).

Quantitative observations of krill in ship-board aquaria (with volumes of $0.06-0.22 \mathrm{~m}^{3}$ ) have indicated that krill hover at a mean angle of approximately $45^{\circ}$ from horizontal (Kils, 1981; Endo, 1993). Kils (1981) also calculated, however, that it is energetically more favorable for krill to swim at speeds of 3-10 $\mathrm{cm} \mathrm{s}^{-1}$ than to hover, due to hydrodynamic lift. Kils (1981) further observed increasingly horizontal orientations at greater swimming speeds, with speeds of $3-10 \mathrm{~cm} \mathrm{~s}^{-1}$ corresponding to angles of $30^{\circ}$ to $<10^{\circ}$ [similar to observations by Miyashita et al. (1996) of E. pacifica]. Our measurements of Antarctic krill orientation are thus consistent with these aquarium studies, if the wild krill are mostly swimming rather than hovering. Supporting this latter notion, observations of $M$. norvegica swimming behavior via acoustic target tracking revealed that this euphausiid swims at a modal speed of ca. $4 \mathrm{~cm} \mathrm{~s}^{-1}$, and there were virtually no observations of stationary individuals (Klevjer and Kaartvedt, 2003).

Krill orientation has also been estimated indirectly from acoustic observations. On the basis of Foote et al.'s (1990) measurements of krill target strengths at 38 and $120 \mathrm{kHz}$ in an experimental enclosure, and using the same theoretical scattering model as employed here, Chu et al. (1993) inferred that the krill oriented on average at $20^{\circ}$ from horizontal $\left[N\left(20^{\circ}, 20^{\circ}\right)\right]$. More recently, Demer and Conti (2005) used a related theoretical scattering model to estimate an orientation distribution of $N\left(15^{\circ}, 5^{\circ}\right)$ from measurements of volume backscattering at 38 and $120 \mathrm{kHz}$ attributed to krill. These results are encouragingly similar to the present observations; the means from both studies fall within less than one standard deviation of the dominant mode of the VPR-derived measurements. In order to infer orientation from observations of volume backscattering or target strength, however, it is necessary to know that the acoustic measurements stem uniquely from krill and not from other scatterers, and all other parameters in the scattering model must be properly constrained. Discrepancies between our measurements of orientation and those from earlier indirect studies may stem from uncertainty in these factors. In the case of the Chu et al.
(1993) study, the estimated distribution of orientations may also have been affected by the krill being in an enclosure where some of them may have been hovering. Our approach to measuring krill orientation is appealing as it captures directly the in situ orientation of the krill in the vicinity of a towed acoustic system. The similarity between our measurements of orientation and the estimates made by Demer and Conti (2005) from a vessel-mounted transducer may suggest that the krill are not substantially disturbed by the passage of the survey vessel.

In using the distribution of krill orientations measured here to parameterize the theoretical krill target strength model, we make two important assumptions. First, we assume that the orientation distribution for the sizes of krill captured by the video system $(\sim 3-15 \mathrm{~mm})$ also applies to larger individuals. Krill start to exhibit schooling behavior at $10 \mathrm{~mm}$ in length, and schools of krill at this size display characteristics identical to those of adults, including uniform orientation and spacing (Hamner et al., 1989). In the absence of any other information, it thus seems reasonable to assume that both large and small krill possess similar schooling behaviors and thereby similar orientations.

Second, we assume that the orientations of the animals observed were not influenced by the presence of the towed body. Hamner and Hamner (2000) found that krill responded to a diver transport vehicle towed at $1 \mathrm{kn}$ by swimming horizontally away, but that tow speeds of 2-4 kn elicited the well-known tail-flip escape response. At a horizontal tow speed of 4-6 kn, and a vertical speed of ca. $0.2 \mathrm{kn}$, any krill able to respond to the BIOMAPER-II towed body before being captured by the video system would be expected to employ the tail-flip response. Many images of krill exhibiting such a response were indeed captured, but excluded from analysis.

Although the dominant mode of krill orientation distribution was centered at $0^{\circ}$, lesser modes were present near $140^{\circ}$ and $-160^{\circ}$. The exact behavior underlying this observation is not known, but it is worth noting that no krill larger than $6 \mathrm{~mm}$ were observed in this "belly-up" orientation (although fewer observations were made of such larger animals). Excluding these smaller modes of angles from the orientation distribution used to parameterize the acoustic scattering model had a negligible effect on predicted target strengths. This is due to the small size of these modes and because a bent cylinder in ventral aspect scatters sound in a very similar fashion to one in dorsal aspect.

The approach to modeling krill scattering employed here represents the krill's shape as a uniformly bent and smoothly tapered cylinder and assumes that the acoustic material properties do not vary within the animal. More sophisticated formulations employ higher resolution shape descriptions to account for appendages and allow for variations in the sound speed and density contrasts along the animal's length (McGehee et al., 1998; Stanton et al., 1998; Stanton and Chu, 2000; Lavery et al., 2002). Similarly, the addition of a stochastic phase component to scattering models has been used to address differences evident at angles away from normal between model predictions of krill scattering and tankbased measurements (Stanton et al., 1998; Demer and Conti, 
2003). When studying the single ping returns from individual animals at discrete angles of orientation (e.g., in the laboratory), the increased accuracy of these more complicated models is desirable. When examining ensembles of animals, as is the case in measurements of volume backscattering strength, and where predicted scattering averaged over a distribution of orientations is dominated by scattering at normal acoustic incidence, errors due to simplifications in the shape description and along-axis variations in material properties become negligible, as do the effects of random phase variability (Stanton and Chu, 2000). Since the animals here are oriented mostly horizontally, and since our interest in knowing krill target strength stems from a desire to make estimates of biologically meaningful quantities from survey measurements of volume backscattering, there is no need to move beyond the lower resolution model nor to include a random phase component. Higher resolution shape models require the digitization of the animal's shape in 2- or 3-D, rather than the simple measurements of length, curvature, and the ratio of length to radius required by the bent cylinder model used here. This latter model thus has the advantage of ease of application (Stanton and Chu, 2000).

Parameterizing the theoretical DWBA-based bent and tapered cylinder model with the distribution of orientations measured here and Chu and Wiebe's (2005) length-based material property regressions resulted in predictions of target strength in relation to length and frequency that for larger krill are consistent with the present in situ empirical observations of krill target strength. The congruence in theoretical predictions and empirical measurements provides support for this model parameterization for the larger animals at least. It is these larger krill that are the subject of the krill fishery and the target of most krill acoustic surveys.

In contrast, in situ observations of the target strength of smaller krill were much higher than predicted. As krill length decreases, the size of the animal's structural features relative to the acoustic wavelength becomes correspondingly smaller, making the model less sensitive to specific choices of parameter values and giving us confidence in its predictions for the small krill. The empirical measurements for these small krill, however, are subject to a number of likely sources of error. For example, they are undoubtedly biased upwards due to their being so near to both the noise and processing thresholds (which on-axis were -94 and $-100 \mathrm{~dB}$ and at the edge of the beam -88 and $-94 \mathrm{~dB}$, for the down- and up-looking 120 $\mathrm{kHz}$ transducers respectively). The density of small krill estimated from the net tows was also higher than for the large krill (Wiebe et al., 2004), increasing the likelihood that multiple targets were erroneously accepted as single targets for these smaller akrill, which would further tend to bias positively the latter's measured target strengths. No animals larger than krill were sampled with the nets, but we also cannot exclude the possibility of confounding sources of scattering such as small zooplankton (e.g., copepods or pteropods) or microstructure. Such scattering would be too weak to be confused with the larger krill target strengths, but might be marginally stronger than the scattering from the small krill, inflating the measurements we are attributing to this size class.

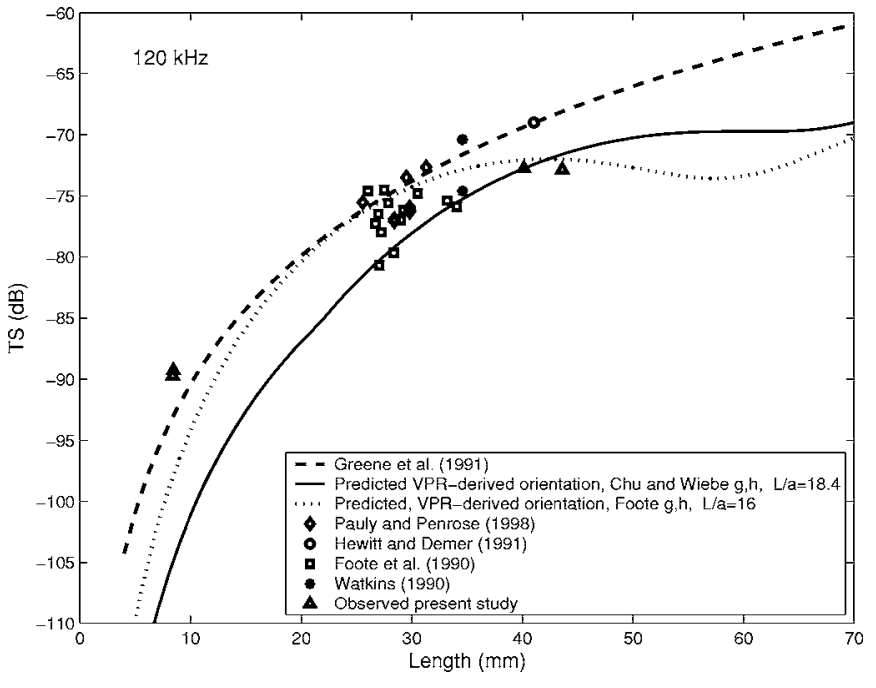

FIG. 8. Target strength at $120 \mathrm{kHz}$ in relation to krill length $(\mathrm{mm})$, showing the Greene et al. (1991) model; the DWBA bent cylinder model parameterized with the present VPR-derived distribution of orientations, Chu and Wiebe's (2005) $g$ and $h$ vs length $(L)$ regressions, and an $L / a_{0}$ of 18.4 (measured for animals in the present study area); and the model parameterized with the VPR-derived distribution of orientations, Foote's (1990) $g$ and $h$ values, and an $L / a_{0}$ of 16 . Also shown are the empirical target strength observations made in the present study, as well as the measurements made by Foote et al. (1990) of krill in an enclosure, by Hewitt and Demer (1991) of krill in situ, and by Pauly and Penrose (1998) of krill in the laboratory, and the target strength estimates made by Watkins (1991) of krill in situ from volume backscattering measurements and photographic estimates of krill density. For the latter study, the plotted points indicate the range of estimated target strength.

Foote et al. (1992) provide a comprehensive review of the then-up-to-date measurements of target strength for a variety of euphausiid species. The Antarctic krill data reviewed were of varying quality, but the measurements of krill target strength at $120 \mathrm{kHz}$ by Foote et al. (1990) and Watkins (1991) were deemed to be of high quality. Since the Foote et al. (1992) review, Hewitt and Demer (1991) and Pauly and Penrose (1998) have also reported observations of krill target strength. Most of these earlier observations lie above our newly parameterized scattering model (Fig. 8). This difference likely stems from two sources. First, experimental error may tend to bias the empirical observations; the Hewitt and Demer (1991) in situ measurements, for instance, are thought to be positively biased by erroneous acceptance of multiple targets as individual target strengths (Demer and Conti, 2005), and the true target strengths for the krill they observed likely fall closer to the predictions of the present model.

Second, there may have existed differences in the exact shape and nutritional status of the different krill populations under investigation, which would require different model parameter values. Our surveys were conducted during austral fall/winter when food resources are low, while previous studies were mostly of krill during summer. The stronger target strengths measured in these earlier studies may relate to the krill being fatter in summer. Properly modeling the target strengths of these summertime krill thus may require a smaller ratio of length to radius $\left(L / a_{0}\right)$ than the value of 18.4 used here. Similarly, the acoustic material properties may vary seasonally: Foote (1990) measured material properties for krill in summer that lead to larger target strength predic- 
tions for the length range of krill he examined than the material property regressions employed here. Indeed, parameterizing the present scattering model with $L / a_{0}=16$ [used previously by Chu et al. (1993) for summertime krill] and Foote's (1990) material property measurements results in predictions of target strength that compare more favorably to the higher values in the range of previous measurements of krill target strength (Fig. 8). This highlights the importance of understanding and measuring wherever possible model parameters appropriate to each particular situation. In the present modeling exercises, all parameters were measured for animals in the actual study region at the time of surveying, and reasonable congruence was achieved between model predictions and empirical observations. Given the low wintertime food conditions experienced by the krill in our study, our target strength predictions should perhaps be taken as a lower bound.

The Greene et al. (1991) semi-empirical model of krill target strength at $120 \mathrm{kHz}$ as a function of length enjoys widespread use in acoustic studies of Antarctic krill. Similar to the findings of earlier studies (McGehee et al., 1998; Demer and Conti, 2003, 2005), the present predictions of krill target strength using the novel model parameterization are at least $4.4 \mathrm{~dB}$ smaller than expected under the Greene et al. (1991) relationship, for all animal lengths investigated. This divergence is particularly strong for small lengths, but Greene et al. (1991) did not intend their model to be used in the Rayleigh scattering region $(k a<1)$. Their model was derived from a linear regression of empirical target strength measurements made at $420 \mathrm{kHz}$ in relation to individual length (Greene et al., 1989; Wiebe et al., 1990). The regression line was then related to anticipated target strengths at the more typical survey frequency of $120 \mathrm{kHz}$, on the theoretical basis of a linearized version of the straight finite cylinder scattering model (Wiebe et al., 1990). This approach assumes a linear relationship between target strength and animal length, although both theoretical and empirical studies indicate that this relationship is nonlinear, due to the complicating influences of animal length, shape, orientation, and material properties. Furthermore, the target strengths of a variety of crustacean species were combined into the target strength to length regression, including the euphausiid $\mathrm{Eu}$ phausia pacifica but not the Antarctic krill itself. Some of the taxa were of quite different body shapes to krill (e.g., decapods and copepods), and the larger body depth to length ratios of these animals may explain much of the difference between the Greene et al. (1991) line and the current model predictions for the relatively thin Antarctic krill.

Greene et al. (1991) recognized that the linearization of the target strength to length relationship constituted a simplification of the scattering process and proposed their model as a practical and highly useful means of estimating krill target strength "until these theoretical models are better developed." Since the Greene et al. (1991) study, DWBA-based approximate theoretical models of zooplankton scattering have progressed considerably and been extensively validated for normal acoustic incidence or averages dominated by normal incidence, especially for euphausiids [reviewed in Stanton and Chu (2000)]. Modern theoretical approaches to mod- eling zooplankton scattering seek to capture the nonlinearities in the target strength versus length relationship, are not limited to any particular frequency (or assumptions concerning the scaling of data from one frequency to another), and include numerous parameters for animal size, shape, orientation, and material properties. These parameters can be adjusted for different taxa, animal behaviors, and body conditions, respectively, thereby providing wide applicability and substantial flexibility. The strong variability in target strength measurements evident in Fig. 8 would suggest that such flexibility is highly desirable.

\section{CONCLUSIONS}

Improved understanding of key model parameters through direct observations of Antarctic krill orientation and application of recently published regressions relating sound speed and density contrasts to krill length has yielded predictions from a theoretical DWBA-based scattering model that compare favorably to in situ measurements of target strength of large krill. The congruence in theoretical predictions and empirical observations provides support for this new model parameterization, as well as further validation of the DWBA-based approach to modeling zooplankton scattering. Arguably, the semi-empirical model of Greene et al. (1991) should be replaced by the use of fully parameterized and field-validated theoretical scattering models like the one developed here, although care must be taken to constrain properly all parameters for the particular krill population at hand. Application of such models will allow more accurate estimates of biologically meaningful quantities like krill abundance and stock biomass from observations of volume backscattering (Everson et al., 1990).

\section{ACKNOWLEDGMENTS}

Chuck Greene provided the initial impetus for this project, when he remarked over lunch that the question of krill target strength had never been fully resolved, and he was amazed that people were still using the Greene et al. (1991) regression model 15 years later. A. Lavery, S. Kaartvedt, Y. Endo, and C. Greene, provided very insightful comments on earlier manuscript drafts. We warmly acknowledge the contributions of C. Davis and S. Gallager, coprimary investigators on the zooplankton broad-scale distribution component of the Southern Ocean GLOBEC project. C. Davis and Q. Hu provided much appreciated help in assessing possible sources of error in the VPR observations. We further thank all officers and crew of the RVIB $N$. B. Palmer; the Raytheon Polar Services Technical Support group; the BIOMAPER-II and MOCNESS teams consisting of C. Davis, M. Dennett, K. Fisher, S. Gallager, A. Girard, E. Horgan, M. Taylor, J. Szelag, and J. Warren; as well as P. Alatalo, M.-Q. Chu, N. Copley, L. Gray, P. Hull, and G. Rosenwaks, for lab analyses of net catches. This project was supported by NSF U.S. Antarctic Program Grant No. OPP9910307. G. Lawson was supported by an Office of Naval Research Graduate Traineeship Award in Ocean Acoustics (Grant No. N000 14-03-1-0212), a Fulbright Scholarship, a Natural Sciences and Engineering Research Council of 
Canada Post-Graduate Scholarship, and the Woods Hole Oceanographic Institution Academic Programs Office. This is U.S. GLOBEC Contribution No. 259.

Anderson, V. C. (1950). "Sound scattering from a fluid sphere," J. Acoust. Soc. Am. 22, 426-431.

Benfield, M. C., Davis, C. S., and Gallager, S. M. (2000). "Estimating the in situ orientation of Calanus finmarchicus on Georges Bank using the Video Plankton Recorder," Plankton Biol. Ecol. 47, 69-72.

Chu, D., and Wiebe, P. H. (2005). "Measurements of acoustic material properties of zooplankton in Antarctic waters," ICES J. Mar. Sci. 62, 818-831.

Chu, D., Foote, K. G., and Stanton, T. K. (1993). "Further analysis of target strength measurements of Antarctic krill at 38 and $120 \mathrm{kHz}$ : Comparison with deformed cylinder model and inference of orientation distribution," J. Acoust. Soc. Am. 93, 2985-2988.

Chu, D., Wiebe, P. H., and Copley, N. (2000). "Inference of material properties of zooplankton from acoustic and resistivity measurements," ICES J. Mar. Sci. 57, 1128-1142.

Davis, C. S., and Wiebe, P. H. (1985). "Macrozooplankton biomass in a warm-core Gulf Stream ring: Time series changes in size structure, taxonomic composition, and vertical distribution," J. Geophys. Res. 90, 88718882.

Davis, C. S., Gallager, S. M., and Solow, A. R. (1992). "Microaggregations of oceanic plankton observed by towed video microscopy," Science $\mathbf{2 5 7}$, 230-232.

Davis, C. S., Gallager, S. M., Marra, M., and Stewart, W. K. (1996). "Rapid visualization of plankton abundance and taxonomic composition using the Video Plankton Recorder," Deep-Sea Res., Part II, 43, 1947-1970.

Demer, D. A., and Conti, S. G. (2003). "Reconciling theoretical versus empirical target strengths of krill: effects of phase variability on the distorted-wave Born approximation," ICES J. Mar. Sci. 60, 429-434.

Demer, D. A., and Conti, S. G. (2005). "New target-strength model indicates more krill in the Southern Ocean," ICES J. Mar. Sci. 62, 25-32.

Endo, Y. (1993). "Orientation of Antarctic krill in an aquarium," Nippon Suisan Gakkaishi 59, 465-468.

Everson, I., Watkins, J. L., Bone, D. G., and Foote, K. G. (1990). "Implications of a new acoustic target strength for abundance estimates of Antarctic krill," Nature (London) 345, 338-339.

Foote, K. G. (1990). "Speed of sound in Euphausia superba," J. Acoust. Soc. Am. 87, 1405-1408.

Foote, K. G., Aglen, A., and Nakken, O. (1986). "Measurement of fish target strength with a split-beam echo sounder," J. Acoust. Soc. Am. 80, 612621.

Foote, K. G., Chu, D., and Stanton, T. K. (1992). "Status of krill target strength," in Selected Scientific Papers, 1992, SC-CAMLR-SSP/9 (Committee for the Conservation of Antarctic Marine Living Resources, Hobart, Australia), pp. 101-126.

Foote, K. G., Everson, I., Watkins, J. L., and Bone, D. G. (1990). "Target strengths of Antarctic krill (Euphausia superba) at 38 and $120 \mathrm{kHz}$," J. Acoust. Soc. Am. 87, 16-24.

Greene, C. H., Wiebe, P. H., and Burczynski, J. (1989). "Analyzing zooplankton size distributions using high-frequency sound," Limnol. Oceanogr. 34, 129-139.

Greene, C. H., Stanton, T. K., Wiebe, P. H., and McClatchie, S. (1991) "Acoustic estimates of Antarctic krill," Nature (London) 349, 110.

Greenlaw, C. F., and Johnson, R. K. (1982). "Physical and acoustical properties of zooplankton," J. Acoust. Soc. Am. 72, 1706-1710.

Hamner, W. M., and Hamner, P. P. (2000). "Behavior of Antarctic krill (Euphausia superba): schooling, foraging, and antipredatory behavior," Can. J. Fish. Aquat. Sci. (Suppl. 3) 57, 192-202.

Hamner, W. M., Hamner, P. P., Obst, B. S., and Carleton, J. H. (1989). "Field observations on the ontogeny of schooling of Euphausia superba furciliae and its relationship to ice in Antarctic waters," Limnol. Oceanogr. 34, 451-456.

Hamner, W. M., Hamner, P. P., Strand, S. W., and Gilmer, R. W. (1983) "Behavior of Antarctic krill, Euphausia superba: Chemoreception, feeding, schooling, and molting," Science 220, 433-435.

Hewitt, R. P., and Demer, D. A. (1991). "Target strength of Antarctic krill," Nature (London) 353, 310

Hofmann, E. E., Klinck, J. M., Costa, D. P., Daly, K. D., Torres, J. J., and Fraser, W. R. (2002). "U.S. Southern Ocean Global Ocean Ecosystem Dynamics Program," Oceanogr. 15, 64-74.

Holliday, D. V., and Pieper, R. E. (1980). "Volume scattering strengths and zooplankton distributions at acoustic frequencies between 0.5 and 3 MHz," J. Acoust. Soc. Am. 67, 135-146.

Ichii, T. (2000). "Krill harvesting," in Krill: Biology, Ecology and Fisheries, edited by I. Everson (Blackwell Science, Oxford), pp. 228-261.

Johnson, R. K. (1977). "Sound scattering from a fluid sphere revisited," J. Acoust. Soc. Am. 61, 375-377.

Kils, U. (1981). "The swimming behavior, swimming performance and energy balance of Antarctic krill, Euphauisa superba," BIOMASS Sci. Ser. No. 3.

Klevjer, T. A., and Kaartvedt, S. (2003). "Split-beam target tracking can be used to study the swimming behavior of deep-living plankton in situ," Aquat. Liv. Res. 16, 293-298.

Kristensen, Å., and Dalen, J. (1986). "Acoustic estimation of size distribution and abundance of zooplankton," J. Acoust. Soc. Am. 80, 601-611.

Lavery, A. C., Stanton, T. K., McGehee, D. E., and Chu, D. (2002). "Threedimensional modeling of acoustic backscattering from fluid-like zooplankton,” J. Acoust. Soc. Am. 111, 1197-1210.

Laws, R. M. (1985). "The ecology of the Southern Ocean," Am. Sci. 73, 26-40.

Lawson, G. L., Wiebe, P. H., Ashjian, C. J., Gallager, S. M., Davis, C. S., and Warren, J. D. (2004). "Acoustically-inferred zooplankton distribution in relation to hydrography west of the Antarctic Peninsula," Deep-Sea Res., Part II, 51, 2041-2072.

Mauchline, J. (1980). "Measurement of body length of Euphausia superba Dana," BIOMASS Handbook No. 4, pp. 4-9.

McGehee, D. E., O’Driscoll, R. L., and Martin-Traykovski, L. V. (1998). "Effects of orientation on acoustic scattering for Antarctic krill at 120 kHz," Deep-Sea Res., Part II, 45, 1273-1294.

Miyashita, K., Aoki, I., and Inagaki, T. (1996). "Swimming behavior and target strength of isada krill (Euphausia pacifica)," ICES J. Mar. Sci. 53, 303-308.

Morse, P. M., and Ingard, K. U. (1968). Theoretical Acoustics (Princeton U. P., Princeton, NJ).

O'Brien, D. P. (1987). "Description of escape responses of krill (Crustacea: Euphausiacea), with particular reference to swarming behavior and the size and proximity of the predator," J. Crust. Biol. 7, 449-457.

Pauly, T., and Penrose, J. D. (1998). "Laboratory target strength measurements of free-swimming Antarctic krill (Euphausia superba)," J. Acoust. Soc. Am. 103, 3268-3280.

Sameoto, D. D. (1980). "Quantitative measurements of euphausiids using a $120 \mathrm{kHz}$ sounder and their in situ orientation," Can. J. Fish. Aquat. Sci. 37, 693-702.

SC-CAMLR (1991). Report of the 10th Meeting of the Scientific Committee (SC-CAMLR-X) (CCAMLR, Hobart, Australia), pp. 117-121.

Stanton, T. K., and Chu, D. (2000). "Review and recommendations for the modeling of acoustic scattering by fluid-like elongated zooplankton: euphausiids and copepods," ICES J. Mar. Sci. 57, 793-807.

Stanton, T. K., Chu, D., and Reeder, D. B. (2004). "Non-Rayleigh acoustic scattering characteristics of individual fish and zooplankton," IEEE J. Ocean. Eng. 29, 260-268.

Stanton, T. K., Chu, D., and Wiebe, P. H. (1998). "Sound scattering by several zooplankton groups. II. Scattering models," J. Acoust. Soc. Am. 103, 236-253.

Stanton, T. K., Chu, D., Wiebe, P. H., and Clay, C. S. (1993). "Average echoes from randomly oriented random-length finite cylinders: Zooplankton models," J. Acoust. Soc. Am. 94, 3463-3472.

Watkins, J. L. (1991). "Krill target strength estimated by underwater photography and acoustics," (WG-Krill-91-40), CCAMLR, Hobart, Australia [as cited in Foote et al. (1992)].

Wiebe, P. H., Greene, C. H., Stanton, T. K., and Burczynski, J. (1990). "Sound scattering by live zooplankton and micronekton: Empirical studies with a dual-beam acoustical system," J. Acoust. Soc. Am. 88, 2346-2360. Wiebe, P. H., Ashjian, C., Gallager, S., Davis, C., Lawson, G. L., and Copley, N. (2004). "Using a High Powered Strobe Light to Increase the Catch of Antarctic Krill," Mar. Biol. (Berlin) 144, 493-502.

Wiebe, P. H., Morton, A. W., Bradley, A. M., Backus, R. H., Craddock, J. E., Cowles, T. J., Barber, V. A., and Flierl, G. R. (1985). "New developments in the MOCNESS, an apparatus for sampling zooplankton and micronekton," Mar. Biol. (Berlin) 87, 313-323.

Wiebe, P. H., Stanton, T. K., Greene, C. H., Benfield, M. C., Sosik, H. M., Austin, T., Warren, J. A., and Hammar, T. (2002). "BIOMAPER II: An integrated instrument platform for coupled biological and physical measurements in coastal and oceanic regimes," IEEE J. Ocean. Eng. 27, 700716. 